\title{
DINÁMICA DE SISTEMAS \\ Y PERSISTENCIA DE NORMAS JURÍDICAS
}

\section{Una mutua incomprensión}

1 estudio del Derecho es susceptible de ser afrontado desde distintos niveles. Por un lado, la llamada dogmática jurídica pretende analizar las normas jurídicas pertenecientes a alguna rama del Derecho previamente delimitada e intenta presentar, a través de ciertas distinciones conceptuales, una visión sistemática de tales normas. Por otro lado, la teoría general del Derecho no analiza las normas de una u otra rama del Derecho, sino los conceptos generales que pueden ser o que de hecho son utilizados por varias de las disciplinas particulares. Si esto es así, pareciera ser que ambos niveles de análisis no son totalmente independientes. Se encontrarían en realidad relacionados en virtud de una escala de abstracción. La dogmática realiza un estudio a un nivel bajo de abstracción, pero debe utilizar ciertos conceptos compartidos por las distintas disciplinas dogmáticas. Estos conceptos generales serían proporcionados por la teoría general del Derecho, puesto que ésta se movería en un nivel más alto de abstracción.

Planteada la cuestión en esos términos, parece que ambos tipos de estudio se necesitan recíprocamente y están condenados a entenderse. Sin embargo, la realidad es algo distinta. Los dogmáticos acusan a los teóricos de proporcionar instrumentos de análisis muy sofisticados, pero inútiles para su cometido; los teóricos acusan a los dogmáticos de no saber aprovechar el instrumental conceptual que la teoría les ofrece.

Lo cierto es que, como suele suceder en estos casos, ambas posiciones tienen su parte de razón. En efecto, en la teoría del Derecho hay la tendencia cada vez más acusada en algunos de sus cultivadores, a lo que podría denominarse la «autogeneración de problemas». Se construye un modelo que intenta, en principio, dar cuenta de algunos problemas que plantea el Derecho y la dogmática. Con el tiempo, el modelo se perfecciona a través de una serie de análisis que aportan cada vez mayores distinciones y una mayor precisión en la formulación. Pero llega un momento en que se corre el peligro de perder el referente y la función originaria del modelo. Se discuten únicamente problemas que genera el modelo, pero ya no los problemas que 
genera el Derecho o la dogmática. Esta circunstancia da pie a que los dogmáticos se distancien de los trabajos de teoría general, puesto que éstos, según ellos, ya no les son útiles. Por otro lado, los teóricos del Derecho entienden que sus estudios ayudarían a conseguir una mayor precisión en la formulación y resolución de los problemas que se plantean los dogmáticos, pero que éstos no los utilizan debido a su complejidad y por el esfuerzo suplementario que se les exigiría. Esta mutua incomprensión genera una dinámica de retroalimentación que amenaza la posibilidad de acuerdo. Así, cuanto más se acusa a la teoría del Derecho de ser inútil para la dogmática más se encierra aquélla en su torre de marfil; cuanto más se acusa a la dogmática de no atender a la teoría del Derecho, más prescinde aquélla de ésta por considerarla cada vez más inútil.

$\mathrm{Si}$ no se desea que esta mutua incomprensión degenere en cortocircuito, son necesarias obras de teoría del Derecho que proporcionen los instrumentos conceptuales necesarios a la dogmática para resolver algunos de sus problemas con mayor precisión y rigor, pero sin olvidar las intuiciones de los dogmáticos, aunque éstas estén formuladas en terminología poco precisa. Al mismo tiempo, se requiere de los dogmáticos una cierta predisposición a ver a la teoría del Derecho como una disciplina que les puede resultar de ayuda, como un aliado más que como un enemigo.

El objetivo de estas páginas es ayudar a romper esta situación dentro de un tema determinado. Intentaré mostrar que con la ayuda de la teoría del Derecho más evolucionada se pueden plantear algunos problemas de la dogmática (en este caso, los relativos a la dinámica de sistemas jurídicos) con mucho más rigor y precisión. Pero, igualmente, procuraré mostrar que para que una teoría del Derecho sea útil a la dogmática es necesario que intente dar cuenta de ciertas intuiciones de la doctrina que, de otro modo, quedarían fuera del modelo teórico. Al final, llegaremos a la conclusión de que alguno de los planteamientos de la teoría del Derecho en este punto pertenece a la categoría de «problemas autogenerados por el modelo».

\section{Dinámica de sistemas jurídicos}

Cuando un jurista se pregunta qué normas deben utilizarse para resolver las controversias jurídicas aparecen ciertas dificultades. Si la respuesta es que se deben utilizar las normas del Derecho nacional de que se trate (por ejemplo, del Derecho español), entonces surge una ambigüedad. Así, una cosa es el Derecho en un momento dado, otra después de una revolución y, otra muy distinta, hablar simplemente del Derecho español. Es obvio que la pregunta que se hace el jurista puede tener respuestas muy distintas según cuál sea el momento en que se formule. Este tipo de cuestiones son las que sirven de marco a la dinámica de sistemas jurídicos. La teoría del Derecho puede ayudar a plantearlas y, en su caso, a resolverlas mediante la clarificación de una serie de conceptos. 


\section{Normas dependientes e independientes}

La reconstrucción sistemática del Derecho requiere postular diferentes clases de normas jurídicas.

Como ha dicho Caracciolo, para construir un sistema normativo $\mathrm{S}$ es preciso indicar una relación R sobre un conjunto de normas, la cual define su estructura. Algunas normas pertenecen al sistema si, y sólo si, satisfacen la relación $\mathrm{R}$ con normas que también pertenecen a S. Son las normas dependientes de S. ${ }^{1}$ Se suelen postular dos relaciones de dependencia. Por un lado, una norma $\mathrm{N}$ es dependiente si es una consecuencia lógica de otra norma N1. Por otro lado, una norma $\mathrm{N}$ es dependiente si la autoridad normativa de $\mathrm{N}$ está autorizada por otra norma N1 a dictar N. Se trata de las relaciones de deducibilidad y de legalidad, respectivamente ${ }^{2}$. Así, deducibilidad y legalidad constituyen criterios de pertenencia de normas dependientes y sirven para definir diferentes tipos de sistemas jurídicos. Aquellos en los que se admite la relación de deducibilidad como condición necesaria y suficiente de pertenencia son sistemas estáticos. Mientras que aquellos en que la relación de legalidad es condición necesaria y suficiente de pertenencia, son sistemas dinámicos. Se suele entender que los sistemas jurídicos son sistemas mixtos. En concreto, de las combinaciones posibles de tales criterios, aquélla en que el criterio de deducibilidad y el de legalidad constituyen condiciones suficientes para la pertenencia, se estima que reconstruye los criterios de pertenencia que los juristas utilizan efectivamente ${ }^{3}$.

Sin embargo, los criterios de deducibilidad y de legalidad son insuficientes para dar cuenta de la pertenencia de todas las normas a un sistema, ya que, si se pretende evitar un regreso al infinito o la circularidad, no cabe que la totalidad de las normas pertenecientes a un sistema satisfagan la relación sistemática. Por eso, las que no la satisfacen pueden denominarse las normas independientes de $\mathrm{S}$, en el sentido de que su incorporación al sistema no depende de la pertenencia de otras normas ${ }^{4}$.

\section{Sistema jurídico, orden jurídico y orden estatal}

La formulación de una norma por una autoridad competente genera un problema respecto a la identidad del sistema jurídico. Puesto que ésta se define extensionalmente, la introducción o eliminación de una norma produce la sustitución de un sistema por otro. No obstante, a pesar de estos cambios, los juristas suelen decir que el sistema jurídico sigue siendo el mismo. Esto

${ }^{1}$ Cfr. Caracciolo, R.: «Sistema jurídico», in Garzón Valdés. E; Laporta F. (eds.): El derecho y la justicia. Enciclopedia iberoamericana de Filosofía. vol. 11, pp. 161-176.

${ }^{2}$ Caracciolo, R.: El sistema jurídico. Problemas actuales, Madrid. C.E.C., 1988, p. 57.

${ }^{3}$ Cfr., además de Caracciolo, ibídem, Bulygin, E.: «Algunas consideraciones sobre los sistemas jurídicos», DOXA, 9, 1991, p. 261. Cfr. también Kelsen, H.: Teoría pura del Derecho (2ª ed.). tr. cast. de R. Tamayo, México, UNAM, 1986, pp. 201-204.

${ }^{4}$ Cfr. Caracciolo, íbid. 
sugiere la pertinencia de realizar la distinción entre sistema jurídico y orden jurídico. Se utiliza la expresión «sistema jurídico» para referirse a conjuntos de normas asociados a un tiempo $\mathrm{t}$ determinado, mientras que se reserva la expresión «orden jurídico» para hacer referencia a una secuencia de sistemas jurídicos. Una norma jurídica pertenece a un sistema jurídico y un sistema jurídico pertenece a un orden jurídico ${ }^{5}$.

Con frecuencia, la distinción de niveles se detiene una vez hechas las anteriores precisiones. Habría, así, para cada momento determinado un conjunto de normas que conformaría un sistema jurídico. Además, distintos sistemas jurídicos asociados a distintos momentos pertenecerían al mismo orden jurídico. Pero, en realidad, en este segundo «nivel», se mezclan comúnmente dos cuestiones, con cuya separación puede ganarse en claridad ${ }^{6}$.

Por ejemplo, a veces se dice que una revolución significa una ruptura del orden jurídico. Y, en ocasiones, se sostiene que, a pesar de los cambios revolucionarios acaecidos en un determinado país, ello no es óbice para considerar que estamos ante el mismo orden jurídico. ¿Cómo pueden compaginarse tales afirmaciones, si es que todas ellas tienen sentido?

Parece de interés proceder a una ulterior distinción entre orden jurídico y orden estatal ${ }^{7}$. Así, se podría afirmar que del mismo modo que un orden jurídico es una sucesión de sistemas jurídicos, un orden estatal es una sucesión de órdenes jurídicos ${ }^{8}$.

${ }^{5}$ Alchourrón, C.; Bulygin, E.: «Sobre el concepto de orden jurídico», in Análisis lógico y Derecho, Madrid, C.E.C., 1991, p. 397.

${ }^{6}$ Sobre este punto ha llamado la atención Caracciolo, pero no ha extraído todas sus consecuencias (Cfr. Caracciolo, R.: Sistema jurídico. Problemas actuales, Madrid, C.E.C., 1988, pp. 19 y 20). Últimamente Bulygin también ha indicado que podría ser útil hacer esta ulterior distinción entre niveles (Bulygin, E.: «Algunas consideraciones sobre los sistemas jurídicos, DOXA, 9, 1991, pp. 265-266).

${ }^{7}$ En muchas ocasiones se incurre en confusiones por no realizar debidamente esta distinción. Entre los muchos ejemplos que podrían traerse a colación, véase Finnis, J. M.: «Revolutions and Continuity of Law», (1973), in Simpson, A. W. B. (ed.): Oxford Essays in Jurisprudence (Second Series), Oxford, O.U.P., 1978. Finnis se da cuenta de la ambigüedad sistemática en que se usa el término «revolución», pero no cae en la cuenta de que él incurre en otro tipo de ambigüedad al usar el mismo término para significar, por un lado, el cambio de orden jurídico, y, por otro, el cambio de orden estatal. Ello sucede cuando, bajo el concepto genérico de «revolución», Finnis procede al análisis del «golpe de Estado» (pp. 46-50), y, a renglón seguido, examina la llamada «sucesión pacífica de Estados» (pp. 52-53). Hay que recordar, al respecto, que suele entenderse por golpe de Estado un intento de cambiar un régimen político dentro de un Estado determinado. De ahí que parezca recomendable tratar el problema de la independencia de un Estado (que afecta al orden estatal) como una cuestión distinta y previa conceptualmente a la de cambio de régimen político (que afecta al orden jurídico).

${ }^{8}$ Para un análisis más detallado de estas cuestiones, cfr. Vilajosana, J.M.: El significado político del derecho. Identidad del orden jurídico y régimen político, México, Fontamara, 1997. Hallar un criterio plausible de identidad de un orden estatal no es nada fácil. La mayoría de las propuestas suelen ser circulares, al contener entre los criterios propiedades normativas. Pero, tal vez, sea posible tomar como criterio el que se suele usar en Derecho internacional: 
Con las distinciones conceptuales precedentes queda claro que pueden plantearse problemas de dinámica de sistemas jurídicos, al menos, a dos niveles distintos. Cuestiones relacionadas, en primer lugar, con el cambio de sistemas jurídicos y, en segundo lugar, con el cambio de órdenes jurídicos. En cada caso, los problemas de dinámica se pueden analizar a partir de dos preguntas: ¿en qué condiciones se producen los cambios de sistema y de orden jurídico? y ¿cómo se explica la persistencia de ciertas normas a pesar de los cambios? Este trabajo se centrará especialmente en la segunda de las preguntas planteadas.

Los sistemas jurídicos, al ser conjuntos de normas, cambian por agregación o por sustracción de normas. La introducción de nuevas normas suele producirse mediante la promulgación, mientras que la eliminación de normas normalmente tiene lugar a través de la derogación'. La doctrina ya ha prestado una atención suficiente a estas cuestiones ${ }^{10}$.

Baste decir que la distinción entre sistema y orden jurídico permite compaginar el cambio de los sistemas jurídicos con la identidad del orden jurídico. El problema básico, sin embargo, es explicar cómo algunas normas que pertenecían a otros sistemas jurídicos anteriores pertenecen también al sistema subsiguiente. Para ello, los juristas suelen postular una cláusula de persistencia:

$\mathrm{CP}$ 1: $\mathrm{Si}$ una norma $\mathrm{Nj}$ pertenece a un sistema jurídico $\mathrm{Sj}$, entonces pertenece a todos los sistemas jurídicos sucesivos del mismo orden jurídico $\mathrm{Oj}$, hasta su eliminación.

En cambio, los problemas que afectan al cambio de orden jurídico han recibido menor atención en la doctrina especializada, por lo que merece la pena detenerse algo más en ellos.

\section{Persistencia de normas a través de órdenes jurídicos}

CPI permite dar cuenta de la dinámica de las normas dentro de un orden jurídico determinado. Sin embargo, en los casos de cambio de orden jurídico (que es común identificar con la ruptura de la legalidad o, como considero preferible, con el cambio de régimen político ${ }^{11}$ ), algunas normas del anterior orden suelen formar parte de los sistemas del nuevo orden jurídico

\footnotetext{
${ }^{9}$ Se deja al margen de este análisis las normas consuetudinarias y aquellas que establecen un ámbito de vigencia temporal predefinido.

${ }^{10}$ Cfr. Alchourrón, C.; Bulygin, E.: «Sobre el concepto de orden jur;dico», in Análisis lógico y Derecho, Madrid, C.E.C., 1991, pp. 398-404; Moreso, J. J.; Navarro, P.: Orden jurídico y sistema jurídico, Madrid, C.E.C., 1993. Aguiló, J.: Sobre la derogación. Ensayo de dinámica jurídica, México, Fontamara, 1995.

${ }^{11}$ Para una discusión acerca de los posibles criterios de identidad de los órdenes jurídicos, cfr. Vilajosana, J.M.: «Towards a material criterion of identity of a legal order» Rechtstheorie, 27, 1996, pp. 45-64.
} 
y, por tanto, la cláusula de persistencia requiere alguna modificación. Una posible formulación sería la siguiente:

$\mathrm{CP}$ 2: $\mathrm{Si}$ una norma $\mathrm{Nj}$ pertenece a un sistema $\mathrm{Sj}$, que es miembro de un orden $\mathrm{O} 1$, entonces $\mathrm{Nj}$ pertenece a todos los sistemas sucesivos a $\mathrm{Sj}$ de todos los órdenes sucesivos a $\mathrm{O} 1$ hasta su eliminación.

Una cláusula de este tipo ha recibido algunas críticas cuya justificación veremos más adelante ${ }^{12}$. Amparándose en esas críticas, y en aras a salvaguardar la concepción sistemática del Derecho, Moreso y Navarro tomarán en consideración la distinción entre pertenencia y aplicabilidad ${ }^{13}$. Si se consideran coextensivos los predicados «pertenece»y «aplicable», no queda otro remedio que intentar reconstruir un criterio de pertenencia de las normas receptadas y así dar cuenta de su admitida fuerza vinculante. En cambio, según estos autores, si se distingue convenientemente ambos predicados, no habría razón para considerarlos coextensivos, con lo cual las normas receptadas, igual que ciertas normas inconstitucionales y ciertas normas extranjeras aplicadas por los tribunales, serían normas aplicables aunque no pertenecientes al sistema jurídico en cuestión.

Así, se acaba formulando lo que podríamos denominar una «cláusula de recepción de normas aplicables»:

CRNA: «Las normas receptadas no son válidas, pero pueden ser aplicables.

Son aplicables si y sólo si otras normas pertenecientes a los nuevos sistemas establecen la obligación o facultad de aplicar esas normas» ${ }^{14}$

Visto el anterior planteamiento, voy a intentar fundamentar mi posición al respecto. En primer lugar, mostraré la necesidad de establecer la identidad de la sucesión de órdenes jurídicos para que pueda hablarse de persistencia de normas entre ellos. Se trata en este caso de una crítica a ambas cláusulas. En segundo lugar, centraré mi atención en diversas críticas a CRNA. Mi estrategia consistirá en poner de relieve que es posible establecer la pertenencia al sistema revolucionario de las normas que persisten. Y ello mediante la propuesta de un criterio de pertenencia al que llamaré «criterio sustantivo de pertenencia». Todo lo cual me permitirá fundamentar una distinción entre persistencia y recepción, así como argumentar que ésta es una mejor construcción que las anteriores por cuanto permite dar cuenta de algunas fuertes y justificadas intuiciones de los juristas.

\section{Identidad de la sucesión}

Tanto CP2 como CRNA plantean algunos problemas a la hora de dar cuenta del carácter dinámico del Derecho.

\footnotetext{
${ }^{12}$ Moreso, J. J.; Navarro, P.E.: «Recepción de normas y sistemas jurídicos», inédito.

${ }^{13}$ Cfr. Bulygin, E.: «Tiempo y validez», in Alchourrón, C; Bul ygin, E.: Análisis lógico y Derecho, Madrid, C.E.C., 1991, pp. 195-214.

${ }^{14}$ Moreso. J.J.; Navarro, P. E.: «Recepción de normas y sistemas jurídicos», cit. p. 19.
} 
Al hablar de «órdenes sucesivos» (CP2) o de «nuevos sistemas» (CRNA) se entiende que se alude a una sucesión de órdenes en el tiempo. Pero veamos qué sucede si sólo contamos con esta propiedad temporal. Imaginemos el caso español. Estaríamos tentados a decir que en el mismo territorio, a partir de un determinado momento, que podemos acordar por hipótesis que sea el de la promulgación de la Constitución de 1978, se ha constituido un nuevo orden jurídico. Sucede, además, que este nuevo orden «recibe» normas del anterior orden jurídico. La pregunta, entonces, puede concretarse de la siguiente forma: ¿Cuál es el criterio que permite afirmar que el orden jurídico inaugurado por la Constitución de 1978 constituye un orden sucesivo respecto al vigente durante el régimen franquista?

Sin hacer una ulterior distinción, sería igualmente posible decantarse como candidato a ser el anterior elemento de la sucesión por cualquier orden jurídico anterior a 1978, con independencia de cuál haya sido el ámbito espacial de validez del mismo. La razón de esta conclusión, que pocos estarían dispuestos a reconocer como una conclusión afortunada, se debe a que en el enunciado de ambas cláusulas sólo se alude al elemento temporal de la sucesión, obviando algo necesario: establecer la identidad de la misma. Si desconocemos que se trata de la sucesión de órdenes jurídicos del mismo orden estatal (o de cualquier otro criterio de identidad que nos parezca relevante), entonces nada obsta para que se escoja como orden jurídico anterior a uno cualquiera perteneciente a un orden estatal distinto. Dicho de otro modo, lo que interesa es determinar la sucesión relevante, ya que existen distintas sucesiones posibles.

Como ya apunté en otro trabajo ${ }^{15}$, el orden que se deben plantear las cuestiones de identidad de los sistemas jurídicos no es caprichoso. Hay una prioridad conceptual de los niveles superiores respecto de los inferiores. Así, hallar o presuponer un criterio de identidad del orden estatal es condición necesaria para resolver los problemas de identidad del orden jurídico. Por esta razón, la cláusula de persistencia (y en su caso la de recepción) debería mencionar la identidad de la sucesión, por ejemplo de este modo:

CP3: $\mathrm{Si}$ una norma $\mathrm{Nj}$ pertenece a un sistema $\mathrm{Sj}$, que es miembro de un orden $\mathrm{O} 1$, perteneciente a un orden estatal $\mathrm{OE}$, entonces $\mathrm{Nj}$ pertenece a todos los sistemas sucesivos a $\mathrm{Sj}$ de todos los órdenes sucesivos a $\mathrm{O} 1$ del orden estatal $\mathrm{OE}$, hasta su eliminación.

De todos modos, alguien podría pensar que se reproducen a nivel de sucesión de órdenes estatales los mismos problemas que, respecto a la sucesión de órdenes jurídicos, CP3 venía a resolver. Pero hay que decir que resulta impropio hablar de «sucesión» de Estados. Como dice Kelsen, «que la totalidad del territorio de un Estado se convierta en territorio de otro es imposible,

${ }^{15}$ Cfr. Vilajosana, J. M.: «Problemas de identidad de los sistemas jurídicos», DOXA, 17-18, 1995, pp. 331-342. 
puesto que, siendo el territorio idéntico, la identidad del Estado subsiste. Así, pues, en tal caso no puede haber sucesión de Estados» ${ }^{16}$.

$\mathrm{Al}$ respecto, hay que advertir que el término «sucesión» es ambiguo, algo que pasa por alto el propio Kelsen. Por un lado, puede utilizarse como sinónimo de ordenación cronológica de elementos o conjuntos. Entonces diríamos, por ejemplo, que el conjunto C2 es sucesivo al conjunto CI, ya que el primero lo asociamos a un momento temporal posterior al segundo, dentro de una misma serie. Éste es el significado que atribuyo a la expresión en el texto. Por otro lado, «sucesivo» también puede significar «sucesor». Éste es el uso que suele predominar en Derecho internacional, cuando se habla de «sucesión de Estados». Un Estado E2 es sucesor del Estado E1 si asume los derechos y las obligaciones contraídas por E1. Pero en este caso, E1 y E2 no tienen por qué formar parte de la misma sucesión (en la primera acepción del término).

Por poner un ejemplo del ámbito familiar, en la sucesión formada por una línea familiar, podría sostenerse que el hijo es el elemento sucesivo al padre, pero no tiene por qué ser el sucesor. Igualmente, la condición de sucesor puede recaer en un individuo que no pertenezca a la misma línea familiar. Si esto es así, en estos casos se puede ser sucesivo sin ser sucesor y sucesor sin ser sucesivo. A nivel estatal, en cambio, si bien no hay sucesión de Estados, nada impide que un Estado determinado, sin ser sucesivo de otro, sea en cambio su sucesor (la República Federal Alemana no es sucesiva respecto a la República Democrática Alemana, en cambio es su sucesora). No realizar esta distinción puede resultar una fuente de confusiones.

Con el fin de ilustrar la idea que quiero subrayar, tómese el caso de desintegración de Checoslovaquia. Podría afirmarse que, en el mismo territorio de lo que antes era Checoslovaquia, a partir de un determinado momento se han constituido dos nuevos órdenes estatales (República Checa y Eslovaquia). Sucede, además, que estos nuevos Estados «recepcionan» normas del Estado checoslovaco. En este caso, ¿cuál sería el criterio que permitiría afirmar que el primer orden jurídico de la República Checa o el primer orden jurídico de Eslovaquia constituyen órdenes sucesivos respecto al último orden jurídico de Checoslovaquia? Se me ocurren tres soluciones:

a) Pensar que se da una persistencia de las normas anteriores y esa persistencia dota de identidad a la sucesión. Esta solución, sin embargo, no es viable. En efecto, nada impedía a la República checa, al empezar su andadura independiente y para evitar el horror vacui, adoptar, por ejemplo, las normas del Derecho español y no las del Estado checoslovaco. Por supuesto, este simple hecho no nos autorizaría a sostener que la República checa es sucesiva del Estado español.

\footnotetext{
${ }^{16}$ Kelsen, H.: Teoría general del Derecho y del Estado, tr. de E. García Máynez, México, UNAM, 1988, p. 273.
} 
b) Pensar que las normas «anteriores» no pertenecen a los nuevos sistemas jurídicos del nuevo orden jurídico del nuevo orden estatal, pero que son aplicables. Si CRNA no se reformula de otra manera, tampoco soluciona el problema, porque de nuevo, el orden jurídico del cual se recepta la norma y el orden jurídico receptante deben pertenecer a la misma sucesión, es decir al mismo orden estatal. Podría pensarse que alguna reformulación de aquella cláusula salvaría este inconveniente; algo que dijera: «... de los ordenes estatales sucesivos». Me temo, sin embargo, que este añadido no soluciona el problema, por cuanto, entonces deberíamos tener un criterio de identidad para decir que E1 y E2 son Estados sucesivos. No bastaría tampoco con la identidad del territorio por cuanto no es el mismo. En definitiva, para sostener que dos elementos son sucesivos hay que contar con un criterio de identidad de la sucesión.

c) Abandonar la idea de recepción en estos casos y distinguirla del concepto de persistencia. La persistencia de normas se daría dentro de un mismo orden estatal, mientras que la recepción quedaría reservada para los casos en que no se puede establecer la identidad de la sucesión.

En este punto es interesante la posición de Kelsen. Este autor sostiene que carece de sentido afirmar que una gran parte del viejo orden jurídico permanece válida dentro del marco del nuevo orden. Únicamente los contenidos de tales normas son los que no cambian, pero sí que cambia la razón de su validez. Si podemos decir que «siguen siendo válidas» bajo la nueva Constitución es sólo porque ésta les confiere validez, expresa o tácitamente. Para Kelsen, el fenómeno es un caso de recepción, y como tal, semejante al que se produjo con la llamada «recepción del Derecho Romano»:

El nuevo orden «recibe», es decir, adopta, normas del viejo orden; esto significa que el nuevo considera válidas normas que poseen el mismo contenido que las del precedente. La «recepción» es un procedimiento abreviado de creación jurídica. La razón de su validez [la de las normas receptadas] no debe buscarse en la Constitución vieja, sino en la nueva, y entre ambas no existe continuidad ${ }^{17}$.

La tesis de Kelsen en este punto no hay que confundirla con CRNA, aunque en ambos casos se hable de recepción. Para Kelsen, a diferencia de CRNA, es impensable la distinción entre normas pertenecientes a un sistema jurídico y normas aplicables. Su postura es más consistente con el uso que los juristas suelen hacer de estas expresiones. En todo caso, si bien la propuesta de Kelsen se ajusta mejor a las intuiciones de los juristas presenta el problema de la ambigüedad de «orden». Efectivamente, al no realizar las distinciones que aquí se han hecho, el término «orden» es empleado a veces para designar a una sucesión de sistemas jurídicos (orden jurídico) y, en otras, como sinónimo de sucesión de órdenes jurídicos (orden estatal).

\footnotetext{
${ }^{17}$ Kelsen, H.: Teoría General del Derecho y del Estado, México, UNAM, 1988, p. 138. Cursiva ajena al original.
} 
Por esa razón, en cualquier caso, se vuelve al punto de partida. Si bien en los cambios de orden revolucionario contamos con un criterio de identidad de la sucesión de órdenes jurídicos, a través del concepto de orden estatal (por tanto sí existe «continuidad», por usar la terminología kelseniana), en los supuestos de «sucesión de Estados» carecemos de un criterio de identidad de la sucesión (aquí sí que es cierto que «no existe continuidad»). Si se admiten estas consideraciones, se podría aceptar que la idea de Kelsen es válida para dar cuenta de la recepción de normas entre órdenes estatales distintos, pero no para la persistencia de normas dentro de un mismo orden estatal. Tendríamos, así, un criterio para distinguir entre los conceptos de persistencia y recepción.

Por tanto, podemos reformular de nuevo la cuestión, circunscribiendo la persistencia de normas a un mismo orden estatal. Ésta es la solución que se va a intentar justificar en el siguiente apartado.

\section{Criterio sustantivo de pertenencia}

Para sostener la plausibilidad de CP3 hay que hacer frente aún a las críticas que la cláusula de persistencia recibe de parte de quienes postulan CRNA. Concentraré mi atención en dos de esas críticas ${ }^{18}$. Ahora me ocuparé de la primera, dejando para el próximo apartado la consideración de la segunda.

La primera crítica consiste en sostener que no se puede aceptar la pertenencia al sistema originario del nuevo orden jurídico de las normas «receptadas» (como se pretende en CP3), puesto que no pueden ser ni normas independientes (ya que presupondrían la pertenencia de otras normas al sistema), ni normas dependientes (ya que no cumplirían con los criterios de deducibilidad o de legalidad).

Lo primero que hay que aclarar al respecto es el distinto carácter de las clasificaciones que aquí están en juego. Mientras la clasificación entre normas independientes y normas dependientes es, por las razones que ya dije en su momento, exhaustiva y necesaria (dentro del contexto sistémico en el que se encuentran las teorías de los sistemas jurídicos), en cambio la división de las normas dependientes entre las que cumplen con el criterio de deducibilidad y el de legalidad no es ni necesaria ni exhaustiva. No hay razones lógicas que lleven a considerar estos dos criterios como necesarios, ni nada hay que impida aportar otros criterios que los complementen. Si los teóricos del Derecho suelen escoger ambos criterios es porque éstos dan cuenta de los usos lingüísticos de los juristas o de cómo funciona un sistema jurídico en la práctica. Efectivamente, parece que los juristas toman en cuenta los criterios de deducibilidad y de legalidad para determinar la pertenencia de las normas dependientes a los sistemas jurídicos vigentes. Pero,

\footnotetext{
${ }^{18}$ Cfr. Moreso. J. J.: Navarro, P.E.: «Recepción de normas y sistemas jurídicos», cit., p. 10.
} 
sin duda, pueden añadirse otros para dar cuenta de otros tantos usos no menos extendidos entre la comunidad jurídica. En este sentido, propongo tomar en consideración un nuevo criterio, al que llamaré «criterio sustantivo de pertenencia» (en adelante, CS).

Una primera formulación de este criterio rezaría como sigue:

CS 1: Una norma $\mathrm{N}$ perteneciente al último sistema jurídico del orden jurídico $\mathrm{O} 1$ pertenece a un sistema jurídico del orden jurídico $\mathrm{O} 2$ del mismo orden estatal OE si es compatible con las normas del primer sistema jurídico de $\mathrm{O} 2$.

Que CS sea un criterio de pertenencia de normas dependientes o independientes no es lo más relevante. La elección entre una u otra opción depende de si se considera que la relación que en él se establece es definitoria de la estructura del sistema o no, es decir, depende de si la postulamos como relación sistemática o extra sistemática. En el primer caso, será un criterio de pertenencia de normas dependientes, y, en el segundo, de normas independientes ${ }^{19}$.

Es más importante, en cambio, destacar que CS no es reducible ni al criterio de legalidad (CL) ni al de deducibilidad (CD). Y ello por dos razones importantes:

1. La norma $\mathrm{N}$, en este caso, no pertenece a un sistema jurídico de la nueva serie porque haya sido dictada por la autoridad competente (más bien, como veremos más adelante, ha sido dictada por una autoridad no competente según la nueva constitución), ni porque sea deducible de las normas independientes del primer sistema del nuevo orden, sino en virtud del criterio sustantivo de pertenencia. $\mathrm{N}$ pertenecía en su momento a un sistema jurídico de la anterior serie por la aplicación de los demás criterios, pero ahora la persistencia se explica por la aplicación de CS.

2. CD, CL y CS son criterios de pertenencia de normas asociados respectivamente a los conceptos de sistema jurídico, orden jurídico y orden estatal. Con ello quiero sugerir lo siguiente:

a) $\mathrm{CD}$ es el único criterio de pertenencia, para cuya aplicación es suficiente tener en cuenta un solo sistema jurídico. Dado un conjunto de normas independientes y por aplicación del CD obtenemos el conjunto de normas dependientes. Es lo que suele denominarse, como ya se dijo, sistema jurídico estático. Por tanto, CD es un criterio de pertenencia de normas al mismo sistema jurídico ${ }^{20}$.

b) La aplicación de CL requiere al menos dos sistemas jurídicos asociados a dos momentos temporales distintos. Por ejemplo, ya no es correcto

${ }^{19}$ Del tenor literal de CS1 se infiere que aquí he escogido tratar a CS como un criterio de pertenencia de normas dependientes, pero, como se dice en el texto, esta elección no tiene mayores implicaciones para el objetivo que aquí se persigue.

${ }^{20}$ Cfr. Alchourrón, C.: Bulygin, E.: Introducción a la metodología de las ciencias jurídicas y sociales, Buenos Aires, Astrea, 1987, p. 92 y ss. Cfr. Caracciolo. R. A.: El sistema jurídico. Problemas actuales, cit.. p. 57. 
afirmar que la norma $\mathrm{N} 2$, que ha sido dictada a tenor del procedimiento establecido por N1 en el tiempo t1, pertenece al mismo sistema S1 que N1. Precisamente la incorporación de $\mathrm{N} 2$ en el tiempo t2 es lo que hace que se pase de S1 a S2. Así, a través de CL tenemos un criterio para dar cuenta del carácter dinámico del Derecho; ahora bien, circunscrito a la serie formada por todos los sistemas jurídicos pertenecientes al mismo orden jurídico O1. Así, pues, N2 pertenece a S2, que es el sistema jurídico sucesivo a S1 dentro de la misma serie (O1). Por tanto, CL es un criterio de pertenencia de normas a distintos sistemas del mismo orden jurídico, o, si se prefiere, un criterio de pertenencia de distintos sistemas al mismo orden jurídico ${ }^{21}$.

c) La aplicación de CS requiere al menos dos órdenes jurídicos asociados a períodos temporales distintos y es pertinente para dar cuenta de la llamada «persistencia del Derecho» dentro de un mismo orden estatal. Ya no es correcto afirmar, por ejemplo, que la norma que persiste (o «receptada») N3 pertenece a un sistema jurídico del nuevo orden por aplicación de CL, puesto que N3 fue promulgada siguiendo un procedimiento no previsto en el nuevo orden jurídico. N3 pertenecía a un sistema jurídico del orden jurídico O1 por la aplicación de $\mathrm{CD}$ o de CL, pero no puede pertenecer a un sistema jurídico perteneciente a $\mathrm{O} 2$ por la aplicación de esos mismos criterios, ya que éstos son criterios de pertenencia de normas a un sistema y de un sistema a un orden jurídico, respectivamente. Lo que se requiere es un criterio como CS, es decir, un criterio de pertenencia de normas a distintos sistemas jurídicos pertenecientes a distintos órdenes jurídicos de un mismo orden estatal. Nótese que CS, a diferencia de CD y de CL, es un criterio de pertenencia que podríamos denominar de «segundo grado», en el sentido de que es operativo sólo respecto de normas que ya han pertenecido a sistemas de la serie anterior por aplicación de los criterios de pertenencia de «primer grado» (CD y CL).

En base a lo anterior, cabe afirmar que CS, a diferencia de CRNA, permite dar cuenta de forma satisfactoria del fenómeno de la persistencia y ello sin apartarnos excesivamente de los usos lingüísticos e intuiciones compartidos por muchos juristas, según los cuales las normas que persisten pertenecen a un sistema del nuevo orden jurídico.

Llegados a este punto, alguien podría objetar que las intuiciones y usos lingüísticos de los juristas no son, sobre esta cuestión, uniformes; por tanto, habría que rechazar la posición que aquí he sostenido por considerarla simplemente una crítica «externa». Sin embargo, creo que es posible añadir una crítica «interna», que como tal puede ser considerada más contundente. Quienes postulan CRNA admiten explícitamente que las normas receptadas no pertenecen a ningún sistema jurídico. Pero, si esto fuera así, tendría como consecuencia claramente implausible el hecho de que las normas

${ }^{21}$ Cfr. Caracciolo, R.: «Sistema jurídico», in Garzón Valdés, E.; Laporta F. (eds.): El derecho y la justicia. Enciclopedia iberoamericana de Filosofía. vol. 11, pp. 161-176. 
receptadas no tendrían carácter jurídico. En efecto, es una tesis compartida por todos los teóricos del Derecho que una norma es jurídica si y sólo si pertenece a un sistema jurídico ${ }^{22}$. Dado que las normas receptadas, si aplicáramos CRNA, no pertenecerían a ningún sistema jurídico, entonces no podrían ser consideradas normas jurídicas. Habría que admitir, en este caso, la conclusión de que sectores enteros del ordenamiento, que todos los juristas sin excepción consideran de carácter jurídico (por ejemplo el código civil español) serían normas aplicables, pero no jurídicas. $\mathrm{Si}$, en cambio, se modifica el criterio de juridicidad de las normas y se admite que es suficiente que alguna vez una norma haya pertenecido a un sistema jurídico para que conserve siempre tal cualidad, entonces aún quedaría en pie lo implausible que resulta sostener que actualmente cuando los jueces aplican el Código civil español, están aplicando normas no pertenecientes al Derecho español. Si no se quiere llegar a alguna de estas conclusiones, hay que abandonar CRNA y circunscribir, si acaso, la recepción de normas aplicables no pertenecientes al orden jurídico de que se trate únicamente a los casos de normas extranjeras (éstas siguen siendo jurídicas porque pertenecen a un sistema jurídico, aunque de un orden estatal distinto). Lo anterior no significa, por supuesto, que la distinción entre pertenencia y aplicabilidad no sea útil para otros casos, tales como la aplicación de normas inconstitucionales ${ }^{23}$.

\section{Incompatibilidad normativa}

La segunda crítica a la que se somete a CP3 y que, por tanto, podría afectar a CS, es la que se refiere a la falta de precisión a la hora de identificar las normas «receptadas». Quienes abogan en favor de CRNA ofrecen como razón de esta imprecisión el hecho de que no exista una noción suficientemente clara de contradicción de normas.

Frente a esta objeción hay que decir lo siguiente. CRNA no soluciona este problema, ya que la misma imprecisión se produce al identificar el conjunto de normas aplicables. En efecto, en CRNA se alude a una norma perteneciente al sistema jurídico postrevolucionario que establece la aplicabilidad de la norma receptada. ¿Cuál puede ser esta norma? De lege ferenda podría postularse la necesidad de que los sistemas jurídicos positivos contengan una norma de este tipo, pero en la práctica pocos la tienen. Parece, más bien, que la candidata idónea a cumplir esta misión sería la disposición derogatoria que normalmente acompaña a la promulgación de una nueva constitución. Esta disposición suele tener un tenor muy general. Por ejemplo, la 95.

${ }^{22}$ Cfr., por ejemplo, Nino, C. S.: Introducción al análisis del Derecho, Barcelona, Ariel, 1983,p.

${ }^{23}$ Véase en este sentido el listado de cuestiones a las que un constitucionalista como Luis María Diez-Picazo aplica una distinción parecida, si bien con distinta terminología. Hay que advertir que el caso de recepción de normas no aparece en el listado. Cfr. Diez-Picazo, L. M.: La derogación de las leyes, Madrid, Civitas, 1990, pp. 167-168. 
Constitución española de 1978, después de derogar explícitamente en los anteriores apartados distintas disposiciones normativas, establece en el apartado 3 de su disposición derogatoria: "Asimismo quedan derogadas cuantas disposiciones se opongan a lo establecido en esta Constitución». Así, cuando se pretenda identificar el conjunto de las normas derogadas aparecerá la misma imprecisión.

El problema específico que presenta la recepción es otro. Se trata de establecer qué significa la «oposición» o la «incompatibilidad» entre normas. Hay, al menos, dos significados posibles, a los que ya alude Kelsen ${ }^{24}$. Por un lado, la incompatibilidad formal y, por otro, la incompatibilidad material. El primer tipo de incompatibilidad es el que presenta, en mi opinión, un problema específico de la persistencia de normas a través de sucesivos órdenes jurídicos del mismo orden estatal. Todas las normas anteriores al nuevo orden jurídico fueron dictadas por órganos que, juzgados a partir de las normas de competencia del nuevo orden jurídico, son incompetentes. No se requiere mostrar la contradicción material, de contenido. Así, por ejemplo, si la Constitución española de 1978 establece que el Parlamento elegido democráticamente y sólo él es competente para dictar leyes, está claro que todas las leyes dictadas por el parlamento anterior no democrático serían inconstitucionales de acuerdo con la nueva constitución, sea cual fuere su contenido.

Si se quiere evitar esta consecuencia, que ningún jurista estaría dispuesto a admitir, hay que postular un criterio para distinguir las normas derogadas de las no derogadas. Pero esto ya se trata de un criterio de interpretación de la disposición derogatoria. A partir de ese momento, ya sólo cabe dar razones extralógicas para diferenciar el conjunto de normas derogadas del conjunto de las normas que siguen perteneciendo al nuevo orden jurídico del mismo orden estatal.

En este sentido, la interpretación que proponen los constitucionalistas es la de atender al contenido y no al procedimiento de creación de las normas anteriores, de tal modo que sólo si se da una contradicción entre el contenido de esas normas y las normas de la Constitución aquéllas quedan derogadas. Valga, por todos, lo dicho por Ignacio de Otto:

(...) la entrada en vigor de la Constitución no trae consigo la derogación de las normas anteriores por el simple hecho de que han sido aprobadas por órganos y procedimientos que la Constitución ha suprimido. Las normas anteriores siguen siendo válidas mientras su contenido no sea contrario a lo que la Constitución dispone, pues de otro modo se produciría la derogación de la totalidad del ordenamiento jurídico. ${ }^{25}$

Ello sugiere una ulterior formulación de CS:

\footnotetext{
${ }^{24}$ Cfr. Kelsen, H.: Teoría pura del derecho, cit., p. 273 y ss.

${ }^{25}$ De Otto, Ignacio: Derecho constitucional. Sistema de fuentes. Barcelona, Ariel, 1987, p. 79.
} 
CS2: Una norma $\mathrm{N}$ perteneciente al último sistema jurídico del orden jurídico $\mathrm{O} 1$ pertenece a un sistema jurídico del orden jurídico $\mathrm{O} 2$ del mismo orden estatal si su contenido es compatible con las normas del primer sistema jurídico de $\mathrm{O} 2$.

La propuesta que aquí he sostenido ofrece, tal como espero haber mostrado, una mayor adecuación a las intuiciones y usos de los juristas y evita consecuencias nada plausibles. Pero posee, además, una ventaja adicional sobre CRNA. No requiere que exista la disposición derogatoria indeterminada en la Constitución. Ello hace que CS2 sirva también para casos como el de la Constitución italiana en los que no existe tal disposición, pero los juristas siguen realizando la misma interpretación. Es cierto que algunos teóricos ${ }^{26}$ opinan que en estos casos cumple la misma función el principio de lex posterior, pero es dudoso que este principio sea de aplicación cuando se ha quebrado su fundamento, que es el criterio de legalidad (y esto es lo que sucede precisamente con el cambio de orden jurídico). Dicho con otras palabras, hecha la distinción entre sistema, orden jurídico y orden estatal, se descubre que el principio de lex posterior encierra una ambigüedad, puesto que puede ir referido al cambio de sistema manteniendo el mismo orden jurídico o al cambio de orden jurídico manteniendo el mismo orden estatal.

A modo de conclusión, cabe decir que, por lo que respecta al tema de la persistencia de las normas jurídicas a través de órdenes jurídicos sucesivos, espero haber mostrado que el problema planteado por algunos teóricos del Derecho no es un problema genuino; se trata, más bien, de un problema autogenerado por el propio modelo. Así, únicamente bajo el presupuesto de que los criterios de pertenencia de las normas dependientes sólo pueden ser el criterio de deducibilidad y el de legalidad se llega a la conclusión de que las normas que persisten no pueden pertenecer a un sistema del nuevo orden jurídico. Sin embargo, una vez modificado el modelo en este punto, no hay mayor inconveniente en ajustar la clasificación a la práctica de los juristas y aceptar un nuevo criterio de pertenencia como CS2. Los constitucionalistas ya habrían dado una solución satisfactoria a la pertenencia de esas normas al sistema postrevolucionario, con lo cual no hay por qué abandonar la concepción sistemática del Derecho (que era la preocupación, esta vez sí genuina, de los teóricos del Derecho), Los dogmáticos, por su parte, harían bien en utilizar las distinciones trazadas por los teóricos del Derecho (por ejemplo, entre sistema, orden jurídico y orden estatal) y de este modo formular de manera más precisa los distintos criterios de pertenencia de normas. He mostrado, además, que cada uno de estos criterios se puede asociar a los distintos niveles en los que a través de aquellas distinciones se puede dividir el tema genérico de la dinámica del Derecho.

${ }^{26}$ Cfr., por todos, Guastini, R.: «Questioni di tecnica legislativa». Le Regioni, 2-3, 1985, pp. 227-228. 
Con lo dicho en este trabajo no espero haber resuelto, ni siquiera planteado, todos los problemas relativos a la dinámica de sistemas jurídicos. Me daría por satisfecho si contribuyo en algo a dictar leyes, está claro que todas las leyes dictadas para erradicar la mutua incomprensión de la que hablé al comienzo y en avanzar en pos de la reconciliación entre dogmática y teoría del derecho. No se me escapa el hecho de que el avance, supuesto que exista, es ciertamente modesto, pero siempre resulta más fructífero dar un pequeño paso en la dirección correcta que recorrer un largo trecho por el camino equivocado. 